\title{
Quantifying Particle Coatings Using High-Precision Mass Measurements
}

\author{
Scott M. Knudsen, ${ }^{\dagger}$ Marcio G. von Muhlen, ${ }^{\dagger, \S}$ and Scott R. Manalis ${ }^{*}, \dagger, \ddagger$ \\ ${ }^{\dagger}$ Departments of Biological Engineering and ${ }^{\ddagger}$ Mechanical Engineering, Massachusetts Institute of Technology, Cambridge, \\ Massachusetts 02139, United States
}

ABSTRACT: We present a general method to quantify coatings on microparticle surfaces based on the additional mass. Particle buoyant mass is determined in a solution with a density that is nearly equivalent to that of the core particle, reducing the magnitude and uncertainty of the measurement. Under these conditions, added material with a different density than that of the core is a larger fraction of the total buoyant mass of the coated particle. This method can resolve a buoyant mass difference between uncoated and coated particles of $\sim 1$ fg. For the protein layer on the $3 \mu \mathrm{m}$ polystyrene spheres measured herein, this is equivalent to $1 / 10$ th of a full layer.
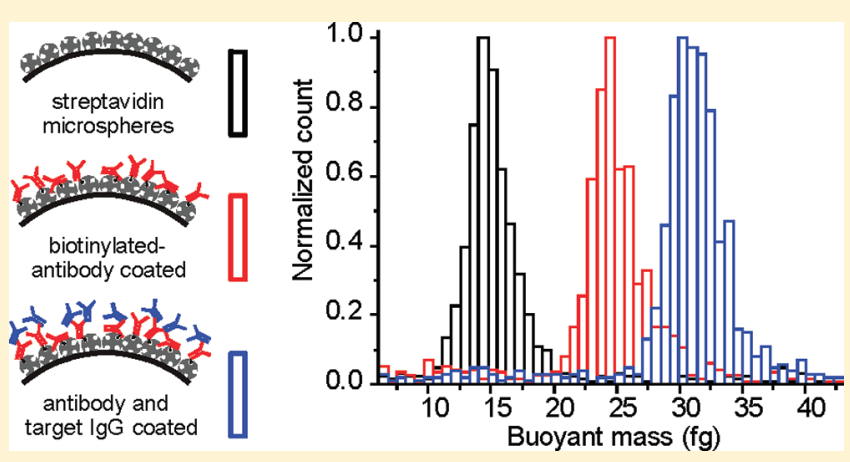

$\mathrm{M}$ icroparticles are currently used in a variety of industrial and biomedical applications and are often coated with different materials to impart functionality for applications such as drug delivery, cell extraction, and biomolecular detection. In many cases, the amount of coating affects the functionality of the particle. Label-based methods such as fluorescence are commonly used for biomolecular detection applications. ${ }^{1-3}$ However, labeling is not always practical and may not be an option in cases where a material layer is added. Although there are a wide range of label-free approaches for measuring the amount of coating on a flat surface, there are very few analogous approaches for particles.

Scanning and transmission electron microscopes can visualize coatings; however, the throughput is limited. ${ }^{4,5} \mathrm{X}$-ray photoelectron spectroscopy can provide an elemental composition of the coating but is not generally suitable for providing an absolute measure of the amount of material. ${ }^{6}$ There are various approaches for measuring the amount of coating provided a specific assumption is made about its properties. For example, the coverage of charged molecules may be quantified based on the $\zeta$ potential of the particle. ${ }^{7}$ If coatings are deposited from solution, it may be possible to determine the amount of material deposited on a batch of particles by quantifying the material in the solute before and after the coating process. ${ }^{8}$ Similarly, conventional gravimetric analysis involves weighing equivalent batches of particles before and after depositing the coating. ${ }^{9}$ However this requires a significant number of particles, is susceptible to unbound contaminants, and depends on an accurate count of the particles measured.

We have previously demonstrated that the suspended microchannel resonator (SMR) can weigh individual microparticles with femtogram precision. ${ }^{10}$ Although this level of precision is sufficient to resolve meaningful differences in coating thicknesses between populations of microparticles, such measurements have remained challenging for two reasons: (i) since the weight of the microparticle is generally many orders of magnitude larger than its coating, variation in particle mass across even the most monodisperse population can obscure the mass of the coating, and (ii) sample-to-sample variations in the density of the carrier solution and density drift during the measurement of an individual sample give rise to significant differences in buoyant mass. Here we address these limitations by adjusting the density of the carrier solution to diminish the buoyant mass of the particle with respect to its coating and by monitoring solution density throughout the measurement using rapid fluid exchanges with a reference solution in an adjacent bypass. This method is appropriate for polymer-based microparticles coated with materials of a different density. For a protein coating on a $3 \mu \mathrm{m}$ polystyrene microsphere, we can resolve approximately $10 \%$ of a full layer.

A particle's buoyant mass depends on its volume and density with respect to the solution density. As the density of the solution approaches the density of the particle, the buoyant mass of the particle approaches zero. Provided the coating density differs from the particle density, it is in principle possible to null out the particle's buoyant mass and weigh only the buoyant mass of the coating. Although it is difficult to exactly match the solution and particle density in practice, even a closely matched solution will improve the precision at which the coating can be resolved. In Figure 1 a population of $3 \mu \mathrm{m}$ diameter polystyrene microspheres is weighed first in water, then in a series of solutions with increasing density. Polystyrene $\left(1.05 \mathrm{~g} / \mathrm{cm}^{3}\right)$ is denser than water, and this population is determined to have a mean buoyant mass of $697 \mathrm{fg}$ with a standard deviation of $7.4 \mathrm{fg}$ (Figure 1, inset a). As the solution

Received: January 4, 2012

Accepted: January 5, 2012

Published: January 5, 2012 


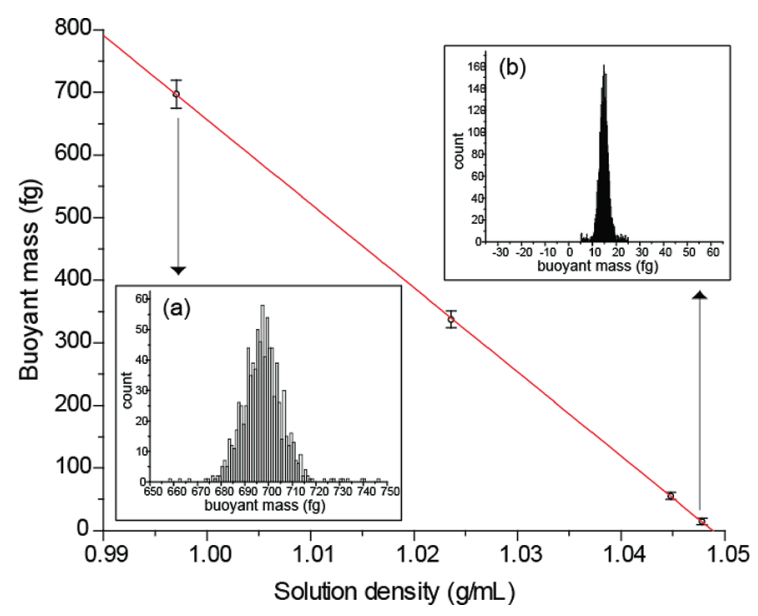

Figure 1. A population of particles in a solution of lesser density will have a positive buoyant mass, with decreasing buoyant mass as the solution density approaches that of the particle. If the particles are of uniform density, the variation in the population will decrease as well. Error bars on the main plot represent $\pm 3 \sigma$, and the two insets are plotted with equivalent $x$-axis scaling.

density is increased by the addition of $\mathrm{D}_{2} \mathrm{O}$ in place of $\mathrm{H}_{2} \mathrm{O}$, both the magnitude and standard deviation of the mean buoyant masses decrease. When the solution density is close to that of the beads, the population has a mean buoyant mass of $14.7 \mathrm{fg}$ and a standard deviation of $1.7 \mathrm{fg}$ (Figure 1, inset b). The limiting factor in determining a small difference in mass between two populations of microparticles is typically based on the standard error of the population mean (SEM). If we assume equivalent sample sizes of $N=1000$ particles, the SEM for these microspheres is $0.23 \mathrm{fg}$ when measured in water and $0.053 \mathrm{fg}$ when measured in an $\mathrm{H}_{2} \mathrm{O} / \mathrm{D}_{2} \mathrm{O}$ mixture at near neutral buoyancy. In the latter fluid, the limit of detection (defined here as $3 \times \mathrm{SEM}$ ) would be a buoyant mass of $0.16 \mathrm{fg}$. If we assume a density of $1.35 \mathrm{~g} / \mathrm{cm}^{3}$ for $150 \mathrm{kDa}$ proteins, ${ }^{11}$ this buoyant mass corresponds to an absolute mass of $2.5 \mathrm{ng} /$ $\mathrm{cm}^{2}$ or $\sim 2900$ molecules per bead. For comparison to routinely used label-free detectors on flat surfaces, commercial surface plasmon resonance (SPR) systems can resolve a smaller mass per area of $0.05 \mathrm{ng} / \mathrm{cm}^{2}$ but require a larger mass of $1000 \mathrm{fg} .{ }^{12}$

To compare the buoyant masses of two or more populations of particles, it is necessary to do so at an equivalent solution density, otherwise buoyant mass differences that arise from differences in solution density may obscure the true mass differences between the samples. For a given set of particles, the slope of a plot similar to Figure 1 provides a scaling factor with which multiple samples can be adjusted to a common solution density. For $3 \mu \mathrm{m}$ diameter particles, a $1 \mathrm{fg}$ change in buoyant mass can be brought about by changing the density of the solution by $7.4 \times 10^{-5} \mathrm{~g} / \mathrm{cm}^{3}$. In comparison, the solution density resolution of the SMR used herein is $1 \times 10^{-6} \mathrm{~g} / \mathrm{cm}^{3}$ when measured with a $1 \mathrm{~s}$ averaging time. Thus if we monitor the solution density as each particle is weighed, variations in buoyant mass can be readily accounted for.

As a particle transits the SMR, it causes a transient shift in the resonant frequency, the maximum of which occurs when the particle is at the apex. ${ }^{10}$ The height of this peak determines the buoyant mass of the particle, while the baseline of the peak (the steady frequency before and after the particle transit) is due to the mass of the solution immediately surrounding the particle. In order to separate changes in this baseline frequency that arise from signal drift from those due to variations in solution density, the SMR is automatically filled with a reference solution of known density (water) at regular intervals, typically every $60 \mathrm{~s}$. A reference baseline is interpolated between each of these frequency measurements, and the difference in frequency between the peak baseline and the reference baseline corresponds to the difference in density between the sample solution and the reference. In this way, each particle measurement comprises a buoyant mass measurement and a solution density measurement.

We demonstrate our method by depositing and quantifying two successive protein layers on polymer microparticles. Streptavidin-coated polystyrene microspheres (Bangs Laboratories; CP01N/9617) are coated with a biotinylated anti-goat IgG antibody for $1 \mathrm{~h}$ and washed 3 times with PBST (1× phosphate buffered saline, $0.2 \%$ Tween-20). Antibodyfunctionalized beads are then incubated with the target protein under the same conditions. Samples are ultimately suspended in a PBST solution containing $\sim 50 \% \mathrm{D}_{2} \mathrm{O}$. The density of this solution is adjusted by adding either PBST or PBST made with $90 \% \mathrm{D}_{2} \mathrm{O}$ as needed. Buoyant mass measurements for each particle are scaled to the average solution density, and the buoyant mass distributions of the unmodified and proteincoated bead populations are shown in Figure 2. The addition of

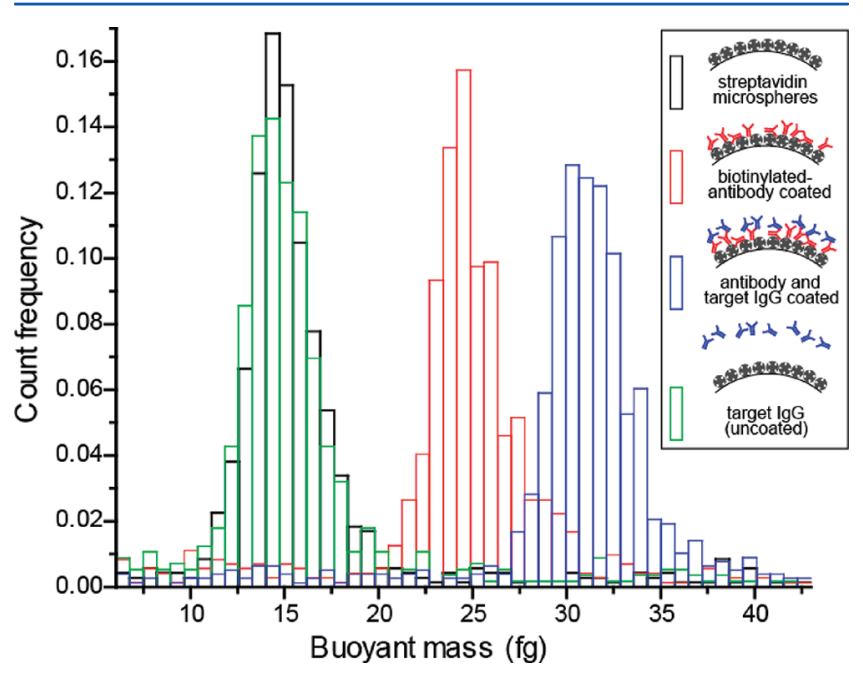

Figure 2. Streptavidin-functionalized polystyrene microspheres are coated with two layers of protein; the first via the streptavidin-biotin interaction and the second via antibody-antigen binding. Particle populations were measured in a solution just below the bulk density of the core microspheres and are plotted at an equivalent solution density. The protein layers add 9.8 and $6.5 \mathrm{fg}$ of buoyant mass, respectively; equivalent to approximately 150 and $100 \mathrm{ng} / \mathrm{cm}^{2}$ absolute mass, assuming a protein density of $1.35 \mathrm{~g} / \mathrm{cm}^{3}$.

the biotinylated antibody layer adds $9.8 \mathrm{fg}$ of buoyant mass at a solution density of $1.055 \mathrm{~g} / \mathrm{mL}$. Assuming a density of 1.35 for protein, this corresponds to $44 \mathrm{fg}$ of absolute mass on a sphere with a $30 \mu \mathrm{m}^{2}$ surface area or $\sim 150 \mathrm{ng} / \mathrm{cm}^{2}$. The antibodyfunctionalized beads can bind a second layer of target protein, in this case goat IgG. Incubation with this protein results in the addition of $6.5 \mathrm{fg}$ buoyant mass, or $\sim 100 \mathrm{ng} / \mathrm{cm}^{2}$. As a control, streptavidin beads that were not antibody-functionalized were incubated with the target IgG used for the second protein layer. As expected, no binding occurs for this sample. In general, the buoyant mass of the material being added to the microparticles could be influenced by the characteristics of the surface 
properties such as its wettability. It is likely that the addition of materials with different surface properties will require individual calibration before the overall buoyant mass of different populations of microparticles can be directly compared.

Although the standard error of the population mean sets the lower limit for resolving added mass, the experimental repeatability takes into account a broader range of errors that may arise in a given system. In order to determine the experimental variation of our system, samples of unmodified beads and beads coated with a layer of biotinylated IgG were measured in triplicate. The standard deviation for each measurement is approximately 2 fg. For 1000 particles, this corresponds to a standard error of 60 ag or a detection limit (3 $\times \mathrm{SEM})$ of $0.18 \mathrm{fg}$. However, experimental variation between equivalent samples is larger than the limit imposed by the standard error. By defining the actual detection limit as 3 times the standard deviation in the mean buoyant mass determined for these triplicate measurements, we achieve limits of detection of 0.66 and $0.9 \mathrm{fg}$ for the uncoated and coated populations, respectively.

We have shown that the addition of a buoyant mass as small as $1 \mathrm{fg}$ to the surface of polystyrene microparticles can be resolved. For the protein layer measured here, this corresponds to $1 / 10$ th of a full layer. However, protein is a relatively light material with a density of $\sim 1.35 \mathrm{~g} / \mathrm{cm}^{3}$. Coatings of denser substances, such as glass or metal would add substantially more mass, and thus a much lower fractional surface coverage could be determined using this method. Conversely, the need to roughly match the density of the carrier liquid to that of the particles places constraints on the core material. A number of so-called heavy liquids have densities suitable for sink-float separation of minerals. For example, an aqueous solution of sodium metatungstate can be in excess of $3.0 \mathrm{~g} / \mathrm{mL}$ at standard temperature and pressure. ${ }^{13}$ These heavy liquids may set the upper limit for the microparticle core density.

\section{AUTHOR INFORMATION}

\section{Corresponding Author}

*E-mail: scottm@media.mit.edu.

\section{Present Address}

${ }^{\S}$ Doximity Inc., San Mateo, CA, 94401, United States.

\section{ACKNOWLEDGMENTS}

We thank Ken Babcock at Affinity Biosensors for valuable discussions. This work was supported by the Institute for Collaborative Biotechnologies through Contract No. W911NF09-D-0001 from the U.S. Army Research Office and Contract No. R01CA119402 from the National Cancer Institute.

\section{REFERENCES}

(1) Krishhan, V. V.; Khan, I. H.; Luciw, P. A. Crit. Rev. Biotechnol. 2009, 29, 29-43.

(2) Kuramitz, H. Anal. Bioanal. Chem. 2009, 394, 61-69.

(3) Ornatsky, O.; Bandura, D.; Baranov, V.; Nitz, M.; Winnik, M. A.; Tanner, S. J. Immunol. Methods 2010, 361, 1-20.

(4) Kim, S. S.; Kim, S. T.; Ahn, J. M.; Kim, K. H. J. Magn. Magn. Mater. 2004, 271, 39-45.

(5) Breen, M. L.; Dinsmore, A. D.; Pink, R. H.; Qadri, S. B.; Ratna, B. R. Langmuir 2001, 17, 903-907.

(6) Meese, T. M.; Hu, Y. H.; Nowak, R. W.; Marra, K. G. J. Biomater. Sci., Polym. Ed. 2002, 13, 141-151.

(7) Toublan, F. J. J.; Boppart, S.; Suslick, K. S. J. Am. Chem. Soc. 2006, 128, 3472-3473.
(8) Jongpoiboonkit, L.; Franklin-Ford, T.; Murphy, W. L. Adv. Mater. 2009, 21, 1960-1963.

(9) Shi, X. Y.; Briseno, A. L.; Sanedrin, R. J.; Zhou, F. M. Macromolecules 2003, 36, 4093-4098.

(10) Burg, T. P.; Godin, M.; Knudsen, S. M.; Shen, W.; Carlson, G.; Foster, J. S.; Babcock, K.; Manalis, S. R. Nature 2007, 446, 1066-1069.

(11) Fischer, H.; Polikarpov, I.; Craievich, A. F. Protein Sci. 2004, 13, $2825-2828$.

(12) Myszka, D. G. Anal. Biochem. 2004, 329, 316-323.

(13) Sahin, M.; Ayranci, K.; Kosun, E.; Ayranci, E. Chem. Geol. 2009, 264, 96-100. 